\title{
Oscillation theorems for second order nonlinear forced differential equations
}

\author{
Ambarka A Salhin", Ummul Khair Salma Din, Rokiah Rozita Ahmad and Mohd Salmi Md Noorani
}

\begin{abstract}
In this paper, a class of second order forced nonlinear differential equation is considered and several new oscillation theorems are obtained. Our results generalize and improve those known ones in the literature.
\end{abstract}

Keywords: Oscillation; Forced nonlinear differential equations of second order

\section{Introduction}

We consider the oscillation behavior of solutions of second order forced nonlinear differential equation

$$
\begin{aligned}
& \left(r(t) \psi(x(t))\left|f\left(x^{\prime}(t)\right)\right|^{\alpha-1} f\left(x^{\prime}(t)\right)\right)^{\prime}+q(t) g(x(t)) \\
& =H\left(t, x(t), x^{\prime}(t)\right), \quad t \in\left[t_{0}, \infty\right)
\end{aligned}
$$

and

$$
\begin{aligned}
& \left(r(t) \psi(x(t)) f\left(x^{\prime}(t)\right)\right)^{\prime}+q(t) g(x(t)) \\
& \quad=H\left(t, x(t), x^{\prime}(t)\right), \quad t \in\left[t_{0}, \infty\right),
\end{aligned}
$$

where $r, q \in C\left(\left[t_{0}, \infty\right), \mathbb{R}\right)$, and $f, \psi, g \in C(\mathbb{R}, \mathbb{R})$ and $H$ is a continuous function on $\left[t_{0}, \infty\right) \times \mathbb{R}^{2}$,

$\alpha$ is a positive real number. Throughout the paper, it is assumed that the following conditions are satisfied:

$$
\begin{aligned}
& \left(\mathrm{A}_{1}\right) r(t)>0, t \geq 0 \\
& \left(\mathrm{~A}_{2}\right) x g(x)>0, g \in C^{1}(\mathbb{R}) \text { for } x \neq 0 \\
& \left(\mathrm{~A}_{3}\right) \frac{H(t, x, y)}{g(x)} \leq p(t) \forall t \in\left[t_{0}, \infty\right) ; x, y \in \mathbb{R} \text { and } x \neq 0 .
\end{aligned}
$$

We restrict our attention only to the solutions of the differential equations (1.1) and (1.2) that exist on some ray $\left[t_{0}, \infty\right)$, where $t_{0} \geq t$, to may depend on the particular solutions. Such a solution is said to be oscillatory if it has arbitrarily large zeros, and otherwise, it is said to be nonoscillatory. Equations (1.1) and (1.2) are called oscillatory if all its solutions are oscillatory.

The problem of finding oscillation criteria for second order nonlinear ordinary differential equations, which involve the average of integral of the alternating coefficient, has received the attention of many authors because in the fact there are many physical systems are modeled by second order nonlinear ordinary differential equations; for example, the so called Emden - Fowler equation arises in the study of gas dynamics and fluid mechanics. This equation appears also in the study of relativistic mechanics, nuclear physics and in the study of chemically reacting systems.

The oscillatory theory as a part of the qualitative theory of differential equations has been developed rapidly in the last decades, and there has been a great deal of work on the oscillatory behavior of differential equations; see e.g. (Agarwal et al. 2010; Beqiri and Koci 2012; Bihari 1963; Elabbasy and Elsharabasy 1997; Elabbasy and Elhaddad 2007; Grace et al. 1984, 1988; Grace and Lalli 1987, 1989, 1990; Grace 1989, 1990, 1992; Greaf and Spikes 1986; Graef et al. 1978; Lee and Yeh 2007; Kamenev 1978; Kartsatos 1968; Li and Agarwal 2000; Meng 1996; Nagabuchi and Yamamoto 1988; Ohriska and Zulova 2004; Ouyang et al. 2009; Philos 1983, 1984, 1985; Remili 2010; Salhin 2014; Tiryaki and Basci 2008; Tiryaki 2009; Temtek and Tiryaki 2013; Yan 1986; Yibing et al. 2013a, b; Zhang and Wang 2010).

Remili (2010), studied the equation

$$
\left(r(t) x^{\prime}(t)\right)^{\prime}+Q(t, x)=H\left(t, x^{\prime}(t), x(t)\right)
$$

and derived some oscillation criteria for the equation (1.3), where new results with additional suitable weighted

* Correspondence: amb80ark@yahoo.com

School of Mathematical Sciences, Faculty of Science and Technology,

Universiti Kebangsaan Malaysia, 43600 UKM Bangi, Selangor, Malaysia 
function are investigated. Zhang and Wang (2010), studied the following equation

$$
\left(r(t) \psi(x(t)) x^{\prime}(t)\right)^{\prime}+Q(t, x)=H\left(t, x^{\prime}(t), x(t)\right) .
$$

Temtek and Tiryaki (2013) obtained several new oscillation results for the equation

$$
\left(r(t) \psi(x(t))\left|x^{\prime}(t)\right|^{\alpha-1} x^{\prime}(t)\right)^{\prime}+Q(t, x)=H\left(t, x^{\prime}(t), x(t)\right),
$$

and its special cases by using generalized Riccati transformation and well known techniques.

In this paper, we continue in this direction the study of oscillatory properties of equations (1.1) and (1.2). The purpose of this paper is to improve and extend the above mentioned results. Our results are more general than the previous results. The relevance of our results becomes clear due to some carefully selected examples.

\section{Main results}

In this section we prove our main results.

Theorem 2.1. Suppose that, conditions $\left(A_{1}\right)-\left(A_{3}\right)$ hold, and

$$
\begin{aligned}
& \frac{g^{\prime}(x)}{\left(\psi(x)|g(x)|^{\alpha-1} \frac{1}{\alpha}\right.} \geq k>0 \text { for all } x \in \mathbb{R} \\
& 0<k_{1} \leq \frac{f(y)}{y} \leq k_{2} \text { for all } y=x^{\prime}(t) \neq 0 .
\end{aligned}
$$

Let $\rho$ be a positive continuously differentiable function over $[T, \infty)$ such that $\rho^{\prime}(t) \geq 0$ over $\left[T_{0}, \infty\right)$;

$$
\begin{aligned}
& \lim _{t \rightarrow \infty} \int_{T_{0}}^{t} \frac{1}{(\rho(s) r(s))^{1 / \alpha d s=\infty,}} \\
& \lim _{t \rightarrow \infty} \sup _{T_{T_{0}}}^{t} Z(s) d s=\infty,
\end{aligned}
$$

where $Z(s)=\rho(s)\left[(q(s)-p(s))-\lambda r(s)\left(\frac{\rho^{\prime}(s)}{\rho(s)}\right)^{\alpha+1}\right]$ and

$$
\lambda=\frac{\alpha}{\alpha+1}\left(k k_{1}\right)^{\alpha+1}
$$

Then all solutions of equation (1.1) are oscillatory.

Proof. Let $x(t)$ be a non-oscillatory solution on $[T, \infty)$, $T \geq T_{0}$ of the equation (1.1). We assume that $x(t)$ is positive on $[T, \infty), T \geq t_{0}$. A similar argument holds for the case when $x(t)$ is negative. Let

$$
w(t)=\frac{\rho(t) r(t) \psi(x(t))\left|f\left(x^{\prime}(t)\right)\right|^{\alpha-1} f\left(x^{\prime}(t)\right)}{g(x(t))}, t \geq T_{0} .
$$

Then differentiating (2.5), (1.1) and take in account assumptions $\left(\mathrm{A}_{1}\right)$ - $\left(\mathrm{A}_{3}\right)$, (2.2) we have

$$
\begin{aligned}
& w^{\prime}(t) \leq-\rho(t)[q(t)-p(t)]+\frac{\rho^{\prime}(t)}{\rho(t)}|w(t)| \\
& -\frac{1}{k_{2}} \frac{\rho(t) r(t) \psi(x(t))\left|f\left(x^{\prime}(t)\right)\right|^{\alpha-1} f^{2}\left(x^{\prime}(t)\right) g^{\prime}(x)}{g^{2}(x)} .
\end{aligned}
$$

In view of (2.1) we conclude that

$$
\begin{aligned}
& w^{\prime}(t) \leq-\rho(t)[q(t)-p(t)] \\
& +\frac{\rho^{\prime}(t)}{\rho(t)}|w(t)|-\frac{k}{k_{2}} \frac{|w(t)|^{\frac{\alpha+1}{\alpha}}}{(\rho(t) r(t))^{1 / \alpha .}}
\end{aligned}
$$

By using the extremum of one variable function it can be proved that

$$
D X-E X^{\frac{\alpha}{\alpha+1}} \leq \frac{\alpha^{\alpha}}{(\alpha+1)^{\alpha+1}} D^{\alpha+1} E^{-\alpha}, D \geq 0, E>0, X \geq 0 .
$$

Now, by applying this inequality we have

$$
\begin{aligned}
w^{\prime}(t) & \leq-\rho(t)[q(t)-p(t)]+\lambda \frac{r(t)\left(\rho^{\prime}(t)\right)^{\alpha+1}}{(\rho(t))^{\alpha}} \\
& =-\rho(t)\left[q(t)-p(t)-\lambda r(t)\left(\frac{\rho^{\prime}(t)}{\rho(t)}\right)^{\alpha+1}\right] .
\end{aligned}
$$

Integrating (2.8) from $T$ to $t$, we get

$$
w(t) \leq w(T)-\int_{T}^{t} \rho(s)\left[q(s)-p(s)-\lambda r(s)\left(\frac{\rho^{\prime}(s)}{\rho(s)}\right)^{\alpha+1}\right] d s, t \geq T \geq T_{0} .
$$

Taking the limit for both sides of (2.9) and using (2.4), we find $w(t) \rightarrow-\infty$. Hence, there exists $T_{1} \geq T$ such that $f\left(x^{\prime}(t)\right)<0 \Rightarrow x^{\prime}(t)<0, \forall t \geq T_{1}$.

Condition (2.4) also implies that $\int_{T}^{\infty} \rho(s)[q(s)-p(s)] d s=\infty$, and there exists $T_{2} \geq T_{1}$ such that

$$
\int_{T_{1}}^{T_{2}} \rho(s)[q(s)-p(s)] d s=0 \text { and } \int_{T_{2}}^{t} \rho(s)[q(s)-p(s)] d s \geq 0, \quad \forall t \geq T_{2} .
$$


Multiplying Eq. (1.1) by $\rho(t)$ and integrating by parts on $\left[T_{2}, t\right]$, we have

$$
\rho(t)\left[r(t) \psi(x(t))\left|f\left(x^{\prime}(t)\right)\right|^{\alpha}\right]^{\prime} \leq-\rho(t) g(x(t))[q(t)-p(t)] .
$$

Now, integrating by parts, we get

$$
\begin{aligned}
& -\rho(t)\left[r(t) \psi(x(t))\left(-f\left(x^{\prime}(t)\right)\right)^{\alpha}\right]+C_{T_{2}} \leq \\
& -\int_{T_{2}}^{t} \rho^{\prime}(s) r(s) \psi(x(s))\left(-f\left(x^{\prime}(s)\right)\right)^{\alpha} d s \\
& -\int_{T_{2}}^{t} \rho(s) g(x(s))[q(s)-p(s)] d s,
\end{aligned}
$$

where

$$
\begin{gathered}
C_{T_{2}}=\frac{\rho\left(T_{2}\right) r\left(T_{2}\right) \psi\left(x\left(T_{2}\right)\right)\left(-f\left(x^{\prime}\left(T_{2}\right)\right)\right)^{\alpha}}{g\left(x\left(T_{2}\right)\right)}>0 . \\
\rho(t)\left[r(t) \psi(x(t))\left(-f\left(x^{\prime}(t)\right)\right)^{\alpha}\right] \geq C_{T_{2}}+g(x(t)) \int_{T_{2}}^{t} \rho(s)[q(s)-p(s)] d s \\
-\int_{T_{2}}^{t} x^{\prime}(s) g^{\prime}(x(s)) \int_{T_{2}}^{s} \rho(u)[q(u)-p(u)] d u d s \\
+\int_{T_{2}}^{t} \rho^{\prime}(s) r(s) \psi(x(s))\left(-f\left(x^{\prime}(s)\right)\right)^{\alpha} d s \leq C_{T_{2}}, \\
\forall t \geq T_{1} .
\end{gathered}
$$

Therefore,

$$
\rho(t)\left[r(t) \psi(x(t))\left(-f\left(x^{\prime}(t)\right)\right)^{\alpha}\right] \geq C_{T_{2}}
$$

From (2.1) and (2.2), we find

$$
\begin{aligned}
& \psi(x(t))\left(-f\left(x^{\prime}(t)\right)\right)^{\alpha} \geq \frac{C_{T_{2}}}{r(t) \rho(t)}, \\
& (\psi(x(t)))^{\frac{1}{\alpha}}\left(-f\left(x^{\prime}(t)\right)\right) \geq\left(\frac{C_{T_{2}}}{r(s) \rho(s)}\right)^{\frac{1}{\alpha}}, \\
& \int_{T_{2}}^{t} k_{2}(\psi(x(s)))^{\frac{1}{\alpha}} x^{\prime}(s) d s \leq \int_{T_{2}}^{t}\left(\frac{-C_{T_{2}}}{r(s) \rho(s)}\right)^{\frac{1}{\alpha}} d s, \\
& \int_{x\left(T_{2}\right)}^{x(t)} k_{2}(\psi(y))^{\frac{1}{\alpha}} d y \leq \int_{T_{2}}^{t}\left(\frac{-C_{T_{2}}}{r(s) \rho(s)}\right)^{\frac{1}{\alpha}} d s .
\end{aligned}
$$

From (2.3) and $0<x(t) \leq x\left(T_{2}\right)$, this implies that

$\int_{x\left(T_{2}\right)}^{x(t)} k_{2}(\psi(y))^{\frac{1}{\alpha}} d y$ is lower bounded, but the right side of it tends to mines infinity. Then, this is a contradiction.

Example 2.2. Consider the following differential equation

$$
\begin{aligned}
& {\left[\frac{1}{t}\left(13 x^{\prime}(t)+\frac{x^{\prime}(t)}{\left(x^{\prime}(t)\right)^{2}+1}\right]^{\prime}+\left(t+\frac{\sin t}{t}\right) x(t)\right.} \\
& =\frac{2 x^{8} \sin t \cos \left(x^{\prime}(t)+1\right)}{\left(x^{7}+1\right) t^{3}}, \quad t \geq \frac{\pi}{2},
\end{aligned}
$$

Evidently, if we take $p(t)=\frac{2}{t^{3}}, \rho(t)=t$ and $\alpha=2$. Then all conditions of Theorem 2.1 are satisfied, hence, all the solutions are oscillatory.

Theorem 2.3. If $\left(A_{1}\right)-\left(A_{3}\right)$, conditions $(2.1)-(2.3)$ hold, and

$$
\int_{T_{0}}^{\infty} \rho(s)[q(s)-p(s)] d s<\infty
$$

$$
\lim _{t \rightarrow \infty} \inf \left[\int_{T}^{t} Z(s) d s\right] \geq 0 \text { for all large } T
$$

$$
\lim _{t \rightarrow \infty} \int_{T_{0}}^{t}\left(\frac{1}{\rho(s) r(s)} \int_{s}^{\infty} Z(u) d\right)^{1 / \alpha d s=\infty}
$$

and

$$
\int_{ \pm \varepsilon}^{ \pm \infty}\left(\frac{\psi(y)}{g(y)}\right)^{\frac{1}{\alpha}} d y<\infty \text { for every } \varepsilon>0 .
$$

Thus all solutions of Eq. (1.1) are oscillatory.

Proof. Let $x(t)$ be a non-oscillatory solution on $[T, \infty)$, $T \geq T_{0}$ of Eq. (1.1). Let us assume that $x(t)$ is positive on $[T, \infty)$ and consider the following three cases for the behavior of $x^{\prime}(t)$.

Case 1: $x^{\prime}(t)>0$ for $T_{1} \geq T$ for some $t \geq T_{1}$; then from (2.10), we obtain

$$
\begin{aligned}
& \int_{T_{1}}^{t} Z(s) d s \leq \frac{r\left(T_{1}\right) \rho\left(T_{1}\right) \psi\left(x\left(T_{1}\right)\right)\left|f\left(x^{\prime}\left(T_{1}\right)\right)\right|^{\alpha-1} f\left(x^{\prime}\left(T_{1}\right)\right)}{g\left(x\left(T_{1}\right)\right)} \\
& -\frac{\rho(t) r(t) \psi(x(t)) f\left(x^{\prime}(t)\right)^{\alpha}}{g(x(t))} .
\end{aligned}
$$

From (2.1) and (2.2), we obtain

$$
\frac{1}{r(t) \rho(t)} \int_{T_{1}}^{t} Z(s) d s \leq \frac{\psi(x(t)) f\left(x^{\prime}(t)\right)^{\alpha}}{g(x(t))}
$$

Hence, for all $t \geq T_{1}$ 


$$
\begin{aligned}
& \left(\frac{1}{r(t) \rho(t)} \int_{T_{1}}^{\infty} Z(s) d s\right)^{1 / \alpha} \leq \frac{\psi(x(t))^{1 / \alpha} f\left(x^{\prime}(t)\right)}{g(x(t))^{1 / \alpha}} \\
& \int_{T_{1}}^{t}\left(\frac{1}{r(s) \rho(s)} \int_{s}^{\infty} Z(u) d u\right)^{1 / \alpha} d s \leq k_{1} \int_{T_{1}}^{t} \frac{\psi(x(s))^{1 / \alpha} x^{\prime}(s)}{g(x(s))^{1 / \alpha}} d s, \\
& \leq k_{1} \int_{x\left(T_{1}\right)}^{\infty}\left(\frac{\psi(y)}{g(y)}\right)^{1 / \alpha} d y .
\end{aligned}
$$

Using (2.13), we obtain

$$
\int_{T_{1}}^{t}\left(\frac{1}{r(s) \rho(s)} \int_{s}^{\infty} Z(u) d u\right)^{1 / \alpha d s<\infty}
$$

which contradicts to the condition (2.13).

Case 2: If $x^{\prime}(t)$ is oscillatory, then there exists a sequence $\left\{\alpha_{n}\right\} \rightarrow \infty$ on $[T, \infty)$ such that $x^{\prime}\left(\alpha_{n}\right)<0$. Let us assume that $N$ is sufficiently large so that

$$
\int_{\alpha_{N}}^{\infty} Z(s) d s \geq 0
$$

Then, from (2.1), (2.2) and (2.7), we have

$$
\begin{gathered}
-C_{\alpha_{N}}-\int_{\alpha_{N}}^{t} Z(s) d s \geq-\frac{\rho(t) r(t) \psi(x(t))\left(-f\left(x^{\prime}(t)\right)\right)^{\alpha}}{g(x(t))} \\
C_{\alpha_{N}}+\int_{\alpha_{N}}^{t} Z(s) d s \leq \frac{\rho(t) r(t) \psi(x(t))\left(-f\left(x^{\prime}(t)\right)\right)^{\alpha}}{g(x(t))}
\end{gathered}
$$

Thus

$$
\begin{aligned}
& \leq \lim _{t \rightarrow \infty} \inf \frac{\rho(t) r(t) \psi(x(t))\left(-f\left(x^{\prime}(t)\right)\right)^{\alpha}}{g(x(t))} \geq C_{\alpha_{N}} \\
& +\lim _{t \rightarrow \infty} \inf \int_{\alpha_{N}}^{t} Z(s) d s>0
\end{aligned}
$$

which contradicts to the assume that $x^{\prime}(t)$ oscillates.

Case 3: Let $x^{\prime}(t)<0$ for $t \geq T_{1}$. Condition (2.11) implies that for any $t_{0} \geq T_{1}$ such that

$$
\int_{t}^{\infty} \rho(s)[q(s)-p(s)] d s \geq 0 \text { for all } t \geq T_{1} \text {. }
$$

The remaining part of the proof is similar to that of Theorem 2.1 then will be omitted.

Example 2.4. Let us consider the following equation

$$
\begin{aligned}
& {\left[t\left(\frac{x^{4}(t)}{x^{4}(t)+1}\right)\left(7 x^{\prime}(t)+\frac{\left(x^{\prime}(t)\right)^{5}}{\left(x^{\prime}(t)\right)^{4}+1}\right)\right]^{\prime}+\frac{1}{t^{3}} x^{3}(t)} \\
& \quad=\frac{x^{3} \cos x \sin 2 x^{\prime}(t)}{t^{4}}, t>1,
\end{aligned}
$$

Evidently, if we take $p(t)=\frac{1}{t^{4}}, \rho(t)=t$ and $\alpha=1$. Then the equation given in Example 2.2 is oscillatory by Theorem 2.2.

Remark 2.1. Condition (2.10) implies that $\int_{T}^{\infty} Z(s) \geq 0$ and $\liminf \inf _{t \rightarrow \infty} \int_{T}^{\infty} Z(s) d s=\int_{T}^{\infty} Z(s) d s$; hence (2.11) takes the form of $\int_{T}^{\infty} Z(s) \geq 0$, for all large $T$.

Remark 2.2. when $\alpha=1, \psi(x(t))=1$ and $f\left(x^{\prime}(t)\right)=x^{\prime}(t)$, Theorem 2.1 and 2.2 reduce to Theorem 1 and 2 Remili (2010) and Theorems 2.1 and 2.3 are obtained by analogy with Theorems 2.1 and 2.2 from (Temtek and Tiryaki 2013).

Theorem 2.5. Assume that

$$
f(y) \geq b y \text { for all } y \in \mathbb{R} \text { and for some constant } b>0,
$$

$$
0<\int_{0}^{ \pm \varepsilon} \frac{\psi(u)}{g(u)} d u<\infty \text { for all } \varepsilon>0
$$

Furthermore, assume that there exist a constant $A$ such that

$$
\begin{aligned}
& \lim _{t \rightarrow \infty} \sup R(t)=A<\infty, \\
& \text { where } R(t)=\int_{t_{0}}^{t} \frac{d s}{r(s)}, \text { and } \\
& \lim _{t \rightarrow \infty} \sup \int_{t_{0}}^{t} \frac{1}{r(s)} \int_{t_{0}}^{s}[q(u)-p(u)] d u d s=\infty .
\end{aligned}
$$

Then the differentia Eq. (1.2) is oscillatory.

Proof. Without loss of generality, let assume that there exists a solution $x(t)$ of (1.2) such that $x(t)>$ 0 on $[T, \infty)$ for some $T \geq t_{0}$. A similar argument holds also for the case when $x(t)<0$. Let $w(t)$ be defined by the Riccati Transformation

$$
w(t)=\frac{r(t) \psi(x(t)) f\left(x^{\prime}(t)\right)}{g(x(t))}, t \geq T .
$$

Derivation this equality we have 


$$
\begin{aligned}
& w^{\prime}(t)=\frac{\left(r(t) \psi(x(t)) f\left(x^{\prime}(t)\right)\right)^{\prime}}{g(x(t))} \\
& -\frac{r(t) \psi(x(t)) f\left(x^{\prime}(t)\right) g^{\prime}(x(t)) x^{\prime}(t)}{g^{2}(x(t))} .
\end{aligned}
$$

This, and (1.2) imply

$$
w^{\prime}(t) \leq p(t)-q(t) \quad t \geq T .
$$

Integrating this inequality from $T$ to $t(\geq T)$, we obtain

$$
w(t) \leq w(T)-\int_{T}^{t}[q(s)-p(s)] d s
$$

By condition (2.14), we get

$$
\begin{aligned}
& b \frac{r(t) \psi(x(t)) x^{\prime}(t)}{g(x(t))} \leq \frac{r(t) \psi(x(t)) f\left(x^{\prime}(t)\right)}{g(x(t))} \\
& \leq w(T)-\int_{T}^{t}[q(s)-p(s)] d s, \quad b>0
\end{aligned}
$$

Integrating the above inequality multiplied by $\frac{1}{r(t)}$ from $T$ to $t(\geq T)$, we have

$$
\begin{aligned}
& b \int_{T}^{t} \frac{\psi(x(s)) x^{\prime}(s)}{g(x(s))} d s \leq \int_{T}^{t} \frac{\psi(x(s)) f\left(x^{\prime}(s)\right)}{g(x(s))} d s \\
& \leq w(T) R(t)-\int_{T}^{t} \frac{1}{r(s)} \int_{T}^{s}[q(u)-p(u)] d u d s .
\end{aligned}
$$

From condition (2.16) and (2.17), we get that

$$
\theta(t)=\int_{T}^{t} \frac{\psi(x(s)) x^{\prime}(s)}{g(x(s))} d s \rightarrow-\infty \text { as } t \rightarrow \infty .
$$

Now, if $x(t) \geq x(T)$ for large $t$ then $\theta(t) \geq 0$, which is a contradiction. Hence for large $t, x(t) \leq x(T)$, so

$$
\theta(t)=-\int_{x(t)}^{x(T)} \frac{\psi(u)}{g(u)} d u>-\int_{0}^{x(T)} \frac{\psi(u)}{g(u)} d u>-\infty
$$

which is again a contradiction. This completes proof the Theorem 2.3.

Theorems 2.6. Suppose that conditions (2.14), (2.15) and (2.16) hold. Furthermore, suppose that, there exist a function $\rho:\left[t_{0}, \infty\right) \rightarrow(0, \infty)$ such that $\rho^{\prime}(\mathrm{t}) \geq 0$ for all $\mathrm{t} \geq$ $\mathrm{t}_{0}$, and

$$
\lim _{t \rightarrow \infty} \sup \int_{t_{0}}^{t} \frac{1}{\rho(s) r(s)}\left(\int_{t_{0}}^{s} \rho(u)[q(u)-p(u)] d u\right) d s=\infty .
$$

Then the differential equation (1.2) is oscillatory.

Proof. Without loss of generality, let assume that there exists a solution $x(t)$ of (1.2) such that $x(t)>$ 0 on $[T, \infty)$ for some $T \geq t_{0}$. Let $w(t)$ be defined by the Riccati Transformation

$$
w(t)=\rho(t) \frac{r(t) \psi(x(t)) f\left(x^{\prime}(t)\right)}{g(x(t))}, t \geq T .
$$

Derivation this equality we have

$$
\begin{array}{r}
w^{\prime}(t)=\frac{\rho(t)\left(r(t) \psi(x(t)) f\left(x^{\prime}(t)\right)\right)^{\prime}}{g(x(t))}+\frac{\rho^{\prime}(t) r(t) \psi(x(t)) f\left(x^{\prime}(t)\right)}{g(x(t))} \\
-\frac{\rho(t) r(t) \psi(x(t)) f\left(x^{\prime}(t)\right) g^{\prime}(x(t)) x^{\prime}(t)}{g^{2}(x(t))} .
\end{array}
$$

This, and (1.2) imply

$$
w^{\prime}(t) \leq-\rho(t)[q(t)-p(t)]+\frac{\rho^{\prime}(t)}{\rho(t)} w(t) .
$$

Hence for all $t \geq T$, we obtain

$$
\int_{T}^{t} \rho(s)[q(s)-p(s)] d s \leq-\int_{T}^{t} \rho(s) \frac{d}{d s}\left(\frac{w(s)}{\rho(s)}\right) d s .
$$

By the Bonnet's Theorem that for each $t \geq T$, there exist a $T_{0} \in[T, t]$ such that

$$
\begin{aligned}
-\int_{T}^{t} \rho(s) \frac{d}{d s}\left(\frac{w(s)}{\rho(s)}\right) d s & =-\rho(t) \int_{T_{0}}^{t}\left(\frac{d}{d s} \frac{w(s)}{\rho(s)}\right) d s \\
& =-\rho(t) \frac{w(t)}{\rho(t)}+\rho(t) \frac{w\left(T_{0}\right)}{\rho\left(T_{0}\right)} \\
-\int_{T}^{t} \rho(s) \frac{d}{d s}\left(\frac{w(s)}{\rho(s)}\right) d s & =-w(t)+B \rho(t) \quad ; B=\frac{w\left(T_{0}\right)}{\rho\left(T_{0}\right)} .
\end{aligned}
$$

By (2.19) and (2.20) we get

$$
\int_{T}^{t} \rho(s)[q(s)-p(s)] d s=-w(t)+B \rho(t) .
$$

Integrating the above inequality multiplied by $\frac{1}{r(t) \rho(t)}$ from $T$ to $t(\geq T)$, we obtain 


$$
\begin{aligned}
& b \int_{T}^{t} \frac{\psi(x(s)) x^{\prime}(s)}{g(x(s))} d s \leq \int_{T}^{t} \frac{\psi(x(s)) f\left(x^{\prime}(s)\right)}{g(x(s))} d s \\
& \leq B R(t)-\int_{T}^{t}\left(\frac{1}{r(s) \rho(s)} \int_{T}^{s} \rho(u)[q(u)-p(u)] d u\right) d s
\end{aligned}
$$

From (2.16) and (2.18), we have

$$
\theta(t)=\int_{T}^{t} \frac{\psi(x(s)) x^{\prime}(s)}{g(x(s))} d s \rightarrow-\infty \text { as } t \rightarrow \infty .
$$

Now, if $x(t) \geq x(T)$ for large $t$, then $\theta(t) \geq 0$, which is a contradiction. Hence for large $t, x(t) \leq x(T)$, so

$$
\theta(t)=-\int_{x(t)}^{x(T)} \frac{\psi(u)}{g(u)} d u>-\int_{0}^{x(T)} \frac{\psi(u)}{g(u)} d u>-\infty
$$

which is again a contradiction. This completes proof the Theorem 2.6.

Example 2.7. Consider the differential equation

$$
\begin{aligned}
& {\left[e^{t} \frac{x^{4}(t)}{x^{4}(t)+1} x^{\prime}(t)\right]^{\prime}+\left(e^{2 t}+\sin t\right) x^{3}(t)} \\
& =\frac{x^{7}(t) \sin t}{\left(1+x^{4}(t)\right)^{2}} \frac{\left(x^{\prime}(t)\right)^{2}}{\left(x^{\prime}(t)\right)^{2}+1}, \quad t \geq 0 .
\end{aligned}
$$

Here,

$$
\begin{aligned}
& r(t)=e^{t}, q(t)=e^{2 t}+\sin t, \psi(x(t))=\frac{x^{4}(t)}{x^{4}(t)+1}, \\
& g(x)=x^{3}, H\left(t, x(t), x^{\prime}(t)\right)=\frac{x^{7}(t) \sin t}{\left(1+x^{4}(t)\right)^{2}} \frac{\left(x^{\prime}(t)\right)^{2}}{\left(x^{\prime}(t)\right)^{2}+1}, \\
& \frac{H\left(t, x(t), x^{\prime}(t)\right)}{g(x)}=\frac{x^{7}(t) \sin t}{\left(1+x^{4}(t)\right)^{2}} \frac{\left(x^{\prime}(t)\right)^{2}}{\left(x^{\prime}(t)\right)^{2}+1} \\
& \times \frac{1}{x^{3}} \leq \sin t=p(t) .
\end{aligned}
$$

So, can note that

$$
\begin{gathered}
\lim _{t \rightarrow \infty} \sup R(t)=\lim _{t \rightarrow \infty} \sup \int_{t_{0}}^{t} \frac{d s}{e^{s}}<\infty, \\
\int_{0}^{ \pm \varepsilon} \frac{u}{\left(u^{2}\right)^{2}+1} d u=\frac{1}{2} \tan ^{-1} \varepsilon^{2}<\infty
\end{gathered}
$$

Let us take $\rho(t)=1$ we have

$$
\begin{aligned}
& \int_{t_{0}}^{t}\left(\frac{1}{r(s) \rho(s)} \int_{t_{0}}^{s} \rho(u)[q(u)-p(u)] d u\right) d s \\
& \quad=\int_{T}^{t}\left(\frac{1}{e^{s}} \int_{T}^{s}\left[e^{2 u}+\sin u-\sin u\right] d u\right) d s=\infty
\end{aligned}
$$

then, Theorem 2.4 ensures that every solution of the equation given oscillates.

\section{Competing interests}

The authors declare that they have no competing interests.

\section{Authors' contributions}

All authors contributed equally to the writing of this paper. All authors read and approved the final manuscript.

\section{Acknowledgement}

This research has been completed with the support of these grants: DIP2012-31, FRGS/2/2013/SG04/UKM/02/3 and FRGS/1/2012/SG04/UKM/01/1.

Received: 4 April 2014 Accepted: 16 May 2014 Published: 18 June 2014

\section{References}

Agarwal RP, Avramescu C, Mustafa OG (2010) On the oscillation theory of a secondorder strictly sublinear differential equation. Can Math Bull 53(2):193-203

Beqiri XH, Koci E (2012) Oscillation criteria for second order nonlinear differential equations. British Journal of Science 6(2):73-80

Bihari I (1963) An oscillation theorem concerning the half linear differential equation of the Second order. Magyar Tud Akad Mat Kutato Int Kozl 8:275-280

Elabbasy EM, Elhaddad WW (2007) Oscillation of second order nonlinear differential equations with damping term. Electron J Qual Theor Differ Equat 25:1-19

Elabbasy EM, Elsharabasy MA (1997) Oscillation properties for second order nonlinear differential equations. Kyungpook Math J 37:211-220

Grace SR (1989) Oscillation theorems for second order nonlinear differential equations with damping. Math Nachr 141:117-127

Grace SR (1990) Oscillation criteria for second order differential equations with damping. J Austral Math Soc (Series A) 49:43-54

Grace SR (1992) Oscillation theorems for nonlinear differential equations of second order. Math Anal And Appl 171:220-241

Grace SR, Lalli BS (1987) On the second order nonlinear oscillations. Bull Inst Math Acad Sinica 15(no. 3):297-309

Grace SR, Lalli BS (1989) Oscillation theorems for second order nonlinear differential equations with a damping term, Comment. Math Univ Carolinae 30(4):691-697

Grace SR, Lalli BS (1990) Integral averaging technique for the oscillation of second order nonlinear differential equations. J Math Anal Appl 149:277-311

Grace SR, Lalli BS, Yeh CC (1984) Oscillation theorems for nonlinear second order differential equations with a nonlinear damping term. SIAM J Math Anal 15:1082-1093

Grace SR, Lalli BS, Yeh CC (1988) Addendum: Oscillation theorems for nonlinear second order differential equations with a nonlinear damping term. SIAM J Math Anal 19(5):1252-1253

Graef JR, Rankin SM, Spikes PW (1978) Oscillation theorems for perturbed non-linear differential equation. J Math Anal Appl 65:375-390

Greaf JR, Spikes PW (1986) On the oscillatory behaviour of solutions of second order nonlinear differential equation. Czech Math J 36:275-284

Kamenev IV (1978) Integral criterion for oscillation of linear differential equations of second order. Math Zametki 23:249-251

Kartsatos AG (1968) On oscillation of nonlinear equations of second order. J Math Anal Appl 24:665-668

Lee CF, Yeh CC (2007) An Oscillation theorems. Appl Math Lett 20:238-240

Li WT, Agarwal RP (2000) Interval oscillation criteria for second order nonlinear equations with damping. Computers Math Applic 40:217-230

Meng FW (1996) An oscillation theorem for second order superlinear differential equations. Ind J Pure Appl Math 27:651-658 
Nagabuchi Y, Yamamoto M (1988) Some oscillation criteria for second order nonlinear ordinary differential equations with damping. Proc Japan Acad 64:282-285

Ohriska J, Zulova A (2004) Oscillation criteria for second order nonlinear differential equation. IM Preprint Series A 10:1-11

Ouyang Z, Zhong J, Zou S (2009) Oscillation criteria for a class of second-order nonlinear differential equations with damping term. Abstr Appl Anal 2009:1-12

Philos CHG (1983) Oscillation of sublinear differential equations of second order. Nonlinear Anal 7(10):1071-1080

Philos CHG (1984) On second order sublinear oscillation. Aequations Math 27:242-254

Philos CHG (1985) Integral averages and second order superlinear oscillation. Math Nachr 120:127-138

Remili M (2010) Oscillation criteria for second order nonlinear perturbed differential equations. Electron J Qual Theor Differ Equat 25:1-11

Salhin AA (2014) Oscillation criteria of second order nonlinear differential equations with variable Coefficients. Discret Dyn Nat Soc 2014:1-9

Temtek P, Tiryaki A (2013) Oscillation criteria for a certain second-order nonlinear perturbed differential equations. Journal of Inequalities and Applications 524:1-12

Tiryaki A (2009) Oscillation criteria for a certain second-order nonlinear differential equations with deviating arguments. Electron J Qual Theor Differ Equat 61:1-11

Tiryaki A, Basci Y (2008) Oscillation theorems for certain even-order nonlinear damped differential equations. Rocky Mt J Math 38(3):1011-1035

Yan J (1986) Oscillation theorems for second order linear differential equations with damping. Proc Amer Math Soc 98(2):276-282

Yibing S, Zhenlai H, Shurong S, Chao Z (2013a) Interval Oscillation Criteria for Second-Order Nonlinear Forced Dynamic Equations with Damping on Time Scales. Abstr Appl Anal 2013:1-11

Yibing S, Zhenlai H, Shurong S, Chao Z (2013b) Fite-Wintner-Leighton-Type Oscillation Criteria for Second-Order Differential Equations with Nonlinear Damping. Abstr Appl Anal 1-10:2012

Zhang Q, Wang L (2010) Oscillatory behavior of solutions for a class of second-order nonlinear differential equation with perturbation. Acta Appl Math 110:885-893

doi:10.1186/2193-1801-3-300

Cite this article as: Salhin et al:: Oscillation theorems for second order nonlinear forced differential equations. SpringerPlus 2014 3:300.

\section{Submit your manuscript to a SpringerOpen ${ }^{\circ}$ journal and benefit from:}

- Convenient online submission

- Rigorous peer review

- Immediate publication on acceptance

- Open access: articles freely available online

- High visibility within the field

- Retaining the copyright to your article

Submit your next manuscript at $>$ springeropen.com 